\title{
Semirelativistic Bound-State Equations: Trivial Considerations
}

\author{
Wolfgang Lucha ${ }^{1, a}$ and Franz F. Schöberl ${ }^{2, b}$ \\ ${ }^{1}$ Institute for High Energy Physics, Austrian Academy of Sciences, Nikolsdorfergasse 18, A-1050 Vienna, \\ Austria \\ ${ }^{2}$ Faculty of Physics, University of Vienna, Boltzmanngasse 5, A-1090 Vienna, Austria
}

\begin{abstract}
Observing renewed interest in long-standing (semi-) relativistic descriptions of two-body bound states, we would like to make a few comments on the eigenvalue problem posed by the spinless Salpeter equation and, illustrated by the examples of the nonsingular Woods-Saxon potential and the singular Hulthén potential, recall elementary tools that, in their quest, practitioners looking for analytic albeit approximate solutions will find useful.
\end{abstract}

\section{Introduction: Spinless Salpeter equation}

Recent years have witnessed a rise of attempts to study bound states by (semi-) relativistic equations of motion, such as the Klein-Gordon equation, the Dirac equation, or (as a straightforward generalization of the Schrödinger equation) the spinless Salpeter equation, with all its merits and drawbacks (consult, for instance, Refs. [1, 2] for details), derived by nonrelativistic reduction (cf., for instance, Refs. [3-5]) of the Bethe-Salpeter equation $[6,7]$. For two particles of (just for notational simplicity) equal masses, $m$, and relative momentum $\boldsymbol{p}$, interacting via a potential $V(\boldsymbol{x})$ depending on their relative coordinate, $\boldsymbol{x}$, the spinless Salpeter equation may be regarded as the eigenvalue equation of the nonlocal Hamiltonian

$$
H \equiv T(\boldsymbol{p})+V(\boldsymbol{x}), \quad T(\boldsymbol{p}) \equiv 2 \sqrt{\boldsymbol{p}^{2}+m^{2}},
$$

incorporating the relativistic kinetic energy, $T(\boldsymbol{p})$. In view of the interest noted, we revisit this equation for central potentials $V(\boldsymbol{x})=V(r), r \equiv|\boldsymbol{x}|$, by recalling (and exploiting) a couple of well-known results. More precisely, in an almost telegraphic style we sketch, in Sect. 2, some issues relevant for relativistic quantum theory and apply the insights gained, in Sects. 3 and 4, to nonsingular and singular potentials.

\section{Approximate solutions: Strict constraints}

\subsection{Existential question: Maximum number of bound states that can be accommodated}

In contrast to the Coulomb potential $V_{\mathrm{C}}(r) \equiv-\kappa / r, \kappa>0$, lots of rather popular potentials (for instance, the Yukawa or the Woods-Saxon potential) admit only a finite number, $N$, of bound states: this number is a crucial characteristic of bound-state problems. For generic (nonrelativistic) Schrödinger operators

$$
H_{\mathrm{NR}} \equiv \frac{\boldsymbol{p}^{2}}{2 \mu}+V(r), \quad \mu>0, \quad V(r) \leq 0,
$$

\footnotetext{
ae-mail: Wolfgang.Lucha@oeaw.ac.at

be-mail: franz.schoeberl@univie.ac.at
} 
with reduced mass $\mu$, the perhaps most easy-to-evaluate upper bound to $N$ is that one by Bargmann [8]:

$$
N \supsetneqq \frac{I(I+1)}{2}, \quad I \equiv 2 \mu \int_{0}^{\infty} \mathrm{d} r r|V(r)| .
$$

For semirelativistic Hamiltonians of the spinless-Salpeter form (1), an upper bound to $N$ is given by [9]

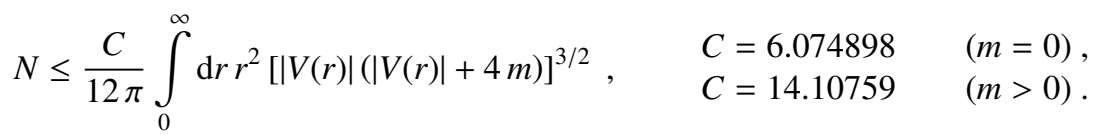

\subsection{Narrowing down solutions: Rigorous bounds on eigenvalues}

As a function of $\boldsymbol{p}^{2}, T(\boldsymbol{p})$ is concave. Thus, $H$ is bounded from above by its Schrödinger limit [10,11]:

$$
H \leq 2 m+\frac{\boldsymbol{p}^{2}}{m}+V(\boldsymbol{x}) \text {. }
$$

The Rayleigh-Ritz variational technique applies to self-adjoint (Hilbert-space) operators, $H$, bounded from below, with eigenvalues $E_{0} \leq E_{1} \leq E_{2} \leq \cdots$ : The d likewise ordered eigenvalues of $H$, restricted to any trial subspace of dimension d of the domain of $H$, form upper bounds to the lowest $d$ eigenvalues of $\mathrm{H}$ below the onset of its essential spectrum. It is favourable to know one's preferred basis of this trial space analytically in both position and momentum spaces. We can achieve this by choosing $[12,13]$ an orthonormal basis defined by means of generalized-Laguerre polynomials $L_{k}^{(\gamma)}(x)$ [14] for parameter $\gamma$, utilizing two variational parameters, $\mu$ (with unit mass dimension) and $\beta$ (which is dimensionless), and spherical harmonics $\mathcal{Y}_{\ell m}(\Omega)$ of angular momentum $\ell$ and projection $m$ depending on the solid angle $\Omega$ :

$$
\begin{aligned}
& \psi_{k, \ell m}(\boldsymbol{x}) \propto r^{\ell+\beta-1} \exp (-\mu r) L_{k}^{(2 \ell+2 \beta)}(2 \mu r) \mathcal{Y}_{\ell m}(\Omega), \\
& L_{k}^{(\gamma)}(x) \equiv \sum_{t=0}^{k}(-1)^{t}\left(\begin{array}{l}
k+\gamma \\
k-t
\end{array}\right) \frac{x^{t}}{t !}, \quad k=0,1,2, \ldots .
\end{aligned}
$$

For the lower end of the spectrum of $H$, the operator inequality $T(\boldsymbol{p}) \geq 2 m \geq 0$ implies $E_{0} \geq \inf _{\boldsymbol{x}} V(\boldsymbol{x})$.

\subsection{Boundedness from below: Constraints on potential parameters}

As an even positive operator, the kinetic-energy term $T(\boldsymbol{p})$ is definitely bounded from below. However, for a potential $V(\boldsymbol{x})$ that is not bounded from below, the issue of the boundedness from below of the full Hamiltonian (1) has to be addressed: The operator $H$ might turn out to be bounded from below only for crucial-potential-parameter values within adequate ranges. For the semirelativistic Coulomb problem, this question has been nicely answered by Herbst a long time ago [15]. In general, this question may be discussed by deriving upper bounds to energy levels, in particular, to the ground state, by using the trial states (6) for quantum numbers $k=\ell=m=0$ and our variational parameter $\beta$ kept fixed at, say, $\beta=1$ :

$$
\psi_{0,00}(\boldsymbol{x}) \propto \exp (-\mu r), \quad \widetilde{\psi}_{0,00}(\boldsymbol{p}) \propto\left(\boldsymbol{p}^{2}+\mu^{2}\right)^{-2} .
$$

\subsection{Accuracy and reliability of solutions: Master virial theorem}

Quality and accuracy $[16,17]$ of an approximate solution to a bound-state equation in use can be easily scrutinized by a relativistic generalization [18] of the virial theorem: All eigenstates \langle\rangle of operators of the form $T(\boldsymbol{p})+V(\boldsymbol{x})$ satisfy a master equation [19] relating the expectation values of radial derivatives:

$$
\left\langle\chi\left|\boldsymbol{p} \cdot \frac{\partial T}{\partial \boldsymbol{p}}(\boldsymbol{p})\right| \chi\right\rangle=\left\langle\chi\left|\boldsymbol{x} \cdot \frac{\partial V}{\partial \boldsymbol{x}}(\boldsymbol{x})\right| \chi\right\rangle \text {. }
$$




\subsection{Desperately seeking analytic results: Seductions and pitfalls}

Aiming at analytic approximations to the exact solutions of spinless Salpeter equations at (almost) any price triggers hectic activity [20-26]: Frequently, close encounters with the nonlocality of the operator $H$ are avoided by expanding $T(\boldsymbol{p})$ up to $O\left(\boldsymbol{p}^{4} / \mathrm{m}^{4}\right)$, to deal with the apparently nicer behaving operators

$$
H_{\mathrm{p}} \equiv 2 m+\frac{\boldsymbol{p}^{2}}{m}-\frac{\boldsymbol{p}^{4}}{4 m^{3}}+V(\boldsymbol{x}) .
$$

However, the expectation value of such "pseudo-spinless-Salpeter Hamiltonian" $H_{\mathrm{p}}$ over, for example, the trial function (8), that is, $\phi(r) \propto \exp (-\mu r)$, reveals that this operator $H_{\mathrm{p}}$ is not bounded from below:

$$
\left\langle H_{\mathrm{p}}\right\rangle=2 m+\frac{\mu^{2}}{m}-\frac{5 \mu^{4}}{4 m^{3}}+\langle V(\boldsymbol{x})\rangle \quad \Longrightarrow \quad \lim _{\mu \rightarrow \infty}\left\langle H_{\mathrm{p}}\right\rangle=-\infty \quad \Longrightarrow \quad E_{0} \leq-\infty .
$$

Consequently, all searches for ground states must be doomed to fail. However, a perturbative approach to $p^{4} / 4 \mathrm{~m}^{3}$, adopted correctly, may save one's day. An expansion over potential-inspired functions [27] mitigates the singularity of the Laplacian's centrifugal term $\propto r^{-2}$, but alters the full effective potential.

\section{Application to potential regular at the origin: Woods-Saxon problem [28]}

The Woods-Saxon (WS) potential is a rather tame potential, familiar from nuclear physics, determined by coupling strength $V_{0}$, potential width $R$, and surface thickness $a$, all of them assumed to be real [29]:

$$
V(r)=V_{\mathrm{WS}}(r) \equiv-\frac{V_{0}}{1+\exp \left(\frac{r-R}{a}\right)}, \quad V_{0}>0, \quad R \geq 0, \quad a>0 .
$$

For definiteness, let's impose the concepts in Sect. 2, as applicable, to the WS eigenvalue problem [28] for the set of mass and potential parameter numerical values of Table 1, dubbed "physical" in Ref. [30]:

- The lower limit to the energy spectrum is, clearly, $E_{0} \geq \inf _{r} V(r)=V(0)=-67.70296 \mathrm{MeV} \gtrsim-V_{0}$.

- The energy interval defined by this bound, $V(0)<E_{k} \leq 0(k=0,1,2, \ldots, N)$, may accommodate for relativistic and nonrelativistic kinematics, respectively, $N \leq 850$ and $N \leq 1201$ eigenstates, at most.

- For (semi-) relativistic WS bound states identified by radial and orbital angular momentum quantum numbers $n_{r}$ and $\ell$, Table 2 presents variational upper bounds to their binding energies derived for our setup $\mu=1 \mathrm{GeV}, \beta=1$, and subspace dimension $d=25$, and the bounds' Schrödinger counterparts.

- The system characterized by the parameter values in Table 1 hardly warrants its relativistic treatment since it is highly nonrelativistic, as the expectation value of $\boldsymbol{p}^{2} / \mathrm{m}^{2}$ over the first trial state (8) reveals:

$$
\left\langle\frac{\boldsymbol{p}^{2}}{m^{2}}\right\rangle \approx 6 \times 10^{-3}
$$

Table 1. Numerical parameter values adopted for the semirelativistic WS problem by the treatment of Ref. [30].

\begin{tabular}{lcccc}
\hline \hline Parameter & $m$ & $V_{0}$ & $R$ & $a$ \\
\hline Numerical value & $940.271 \mathrm{MeV}$ & $67.70352 \mathrm{MeV}$ & $7.6136 \mathrm{fm}$ & $0.65 \mathrm{fm}$ \\
\hline \hline
\end{tabular}


Table 2. Variational and nonrelativistic upper bounds to semirelativistic WS binding energies (in units of GeV).

\begin{tabular}{cccc}
\hline \hline$n_{r}$ & $\ell$ & Spinless Salpeter equation & Schrödinger equation \\
\hline 0 & 0 & -0.06032 & -0.06030 \\
& 1 & -0.05309 & -0.05305 \\
1 & 0 & -0.04119 & -0.04108 \\
& 1 & -0.02967 & -0.02946 \\
2 & 0 & -0.01527 & -0.01545 \\
& 1 & -0.00233 & -0.00362 \\
\hline \hline
\end{tabular}

\section{Application to a potential singular at the origin: Hulthén problem}

The short-range Hulthén potential is characterized by two parameters, coupling strength $v$ and range $b$ :

$$
V(r)=V_{\mathrm{H}}(r) \equiv-\frac{v}{\exp (b r)-1}, \quad b>0, \quad v \geq 0 .
$$

To facilitate comparability, we phrase our remarks for the parameter values used by Ref. [31] (Table 3):

- From the expectation value of the Hamiltonian (1) with Hulthén potential (14) over the trial state (8) we learn that its boundedness from below requires the potential parameters to satisfy $v / b<16 /(3 \pi)$.

- Iff $\kappa \geq v / b$, Hulthén's potential (14) is bounded from below by any Coulomb potential $V_{\mathrm{C}}(r) \equiv-\kappa / r$. Thus, lower bounds to the relativistic Coulomb problem, such as the one given by Herbst [15], apply.

- For a Schrödinger operator (2) with Hulthén potential (14), the eigenvalues for $\ell=0$ states read [32]

$$
E_{n}=-\frac{\left(2 \mu v-n^{2} b^{2}\right)^{2}}{8 \mu n^{2} b^{2}}, \quad n=1,2,3, \ldots, \quad n^{2} b^{2} \leq 2 \mu v .
$$

- Table 4 lists, for $\ell=0$ states, the upper bounds to (semi-) relativistic Hulthén binding energies found variationally for $\mu=1, \beta=1$, and $d=25$, or represented by the analytically given eigenvalues (15).

Table 3. Numerical parameter values adopted for the semirelativistic Hulthén problem in the study of Ref. [31].

\begin{tabular}{lccc}
\hline \hline Parameter & $m$ & $b$ & $v$ \\
\hline Numerical value (arbitrary units) & 1 & 0.15 & 0.11 \\
\hline \hline
\end{tabular}

Table 4. Variational and Schrödinger upper bounds to semirelativistic Hulthén binding energies (arbitrary units).

\begin{tabular}{cccc}
\hline \hline$n_{r}$ & $\ell$ & Spinless Salpeter equation & Schrödinger equation \\
\hline 0 & 0 & -0.10577 & $-0.085069 \dot{4}$ \\
1 & 0 & -0.0022398 & $-0.00 \dot{1}$ \\
\hline \hline
\end{tabular}




\section{Summary and conclusions}

Even though the spinless Salpeter equation resists to being solved by analytical techniques, a variety of elementary considerations allows us to draw a pretty clear picture of the solutions to be expected out of such efforts. Nevertheless, not all solutions offered in the literature do respect the frame of this picture.

\section{References}

[1] W. Lucha and F. F. Schöberl, Int. J. Mod. Phys. A 14, 2309 (1999), arXiv:hep-ph/9812368.

[2] W. Lucha and F. F. Schöberl, Fizika B 8, 193 (1999), arXiv:hep-ph/9812526.

[3] W. Lucha and F. F. Schöberl, Int. J. Mod. Phys. A 7, 6431 (1992).

[4] W. Lucha and F. F. Schöberl, in Proceedings of the International Conference on Quark Confinement and the Hadron Spectrum, edited by N. Brambilla and G. M. Prosperi (World Scientific, River Edge, NJ, 1995), p. 100, arXiv:hep-ph/9410221.

[5] W. Lucha and F. F. Schöberl, Recent Res. Dev. Phys. 5, 1423 (2004), arXiv:hep-ph/0408184.

[6] E. E. Salpeter and H. A. Bethe, Phys. Rev. 84, 1232 (1951).

[7] E. E. Salpeter, Phys. Rev. 87, 328 (1952).

[8] V. Bargmann, Proc. Natl. Acad. Sci. USA 38, 961 (1952).

[9] I. Daubechies, Commun. Math. Phys. 90, 511 (1983).

[10] W. Lucha, F. F. Schöberl, and D. Gromes, Phys. Rep. 200, 127 (1991).

[11] W. Lucha and F. F. Schöberl, Phys. Rev. A 54, 3790 (1996), arXiv:hep-ph/9603429.

[12] S. Jacobs, M. G. Olsson, and C. Suchyta III, Phys. Rev. D 33, 3338 (1986); 34, 3536(E) (1986).

[13] W. Lucha and F. F. Schöberl, Phys. Rev. A 56, 139 (1997), arXiv:hep-ph/9609322.

[14] M. Abramowitz and I. A. Stegun (eds.), Handbook of Mathematical Functions (Dover, New York, 1964).

[15] I. W. Herbst, Commun. Math. Phys. 53, 285 (1977); 55, 316 (1977) (addendum).

[16] W. Lucha and F. F. Schöberl, Phys. Rev. A 60, 5091 (1999), arXiv:hep-ph/9904391.

[17] W. Lucha and F. F. Schöberl, Int. J. Mod. Phys. A 15, 3221 (2000), arXiv:hep-ph/9909451.

[18] W. Lucha and F. F. Schöberl, Phys. Rev. Lett. 64, 2733 (1990).

[19] W. Lucha, Mod. Phys. Lett. A 5, 2473 (1990).

[20] S. M. Ikhdair and R. Sever, Z. Phys. C 56, 155 (1992).

[21] S. M. Ikhdair and R. Sever, Z. Phys. C 58, 153 (1993).

[22] S. M. Ikhdair and R. Sever, Int. J. Mod. Phys. A 19, 1771 (2004), arXiv:hep-ph/0310295.

[23] S. M. Ikhdair and R. Sever, Int. J. Mod. Phys. A 20, 6509 (2005), arXiv:hep-ph/0406005.

[24] S. M. Ikhdair and R. Sever, Int. J. Mod. Phys. E 17, 1107 (2008).

[25] S. Hassanabadi, M. Ghominejad, B. H. Yazarloo, S. Zarrinkamar, and H. Hassanabadi, Chin. Phys. C 37, 083102 (2013).

[26] H. Feizi, M. Hoseininaveh, and A. H. Ranjbar, Int. J. Mod. Phys. E 22, 1350039 (2013).

[27] C. L. Pekeris, Phys. Rev. 45, 98 (1934).

[28] W. Lucha and F. F. Schöberl, Int. J. Mod. Phys. A 29, 1450057 (2014), arXiv:1401.5970 [hep$\mathrm{ph}$.

[29] R. D. Woods and D. S. Saxon, Phys. Rev. 95, 577 (1954).

[30] M. Hamzavi, S. M. Ikhdair, and A. A. Rajabi, Chin. Phys. C 37, 063101 (2013), arXiv:1208.5337 [nucl-th].

[31] S. Zarrinkamar, A. A. Rajabi, H. Hassanabadi, and H. Rahimov, Phys. Scr. 84, 065008 (2011).

[32] S. Flügge, Practical Quantum Mechanics (Springer, Berlin, 1994), Vol. I, pp. 175-178. 
